\title{
Avaliação sobre a adesão às práticas preventivas contra a COVID-19 no cenário do agronegócio na Região do Araguaia no Estado do Pará
}

\author{
Evaluation of adherence to preventive practices against COVID-19 in the agribusiness scenario in \\ the Araguaia Region in the State of Pará \\ Evaluación de la adherencia a prácticas preventivas contra COVID-19 en el escenario \\ agroindustrial en la Región Araguaia en el Estado de Pará
}

Recebido: 31/07/2021 | Revisado: 07/08/2021 | Aceito: 09/08/2021 | Publicado: 14/08/2021

\author{
Raniell Ribeiro da Silva \\ ORCID: https://orcid.org/0000-0001-5309-8783 \\ Faculdade de Ensino Superior da Amazônia Reunida, Brasil \\ E-mail: raniel_qm2004@hotmail.com \\ Mateus Silva Santos \\ ORCID: https://orcid.org/0000-0001-9392-4947 \\ Universidade Federal de Goiás, Brasil \\ E-mail: biomateus07@ outlook.com
}

\begin{abstract}
Resumo
O coronavírus causador da COVID-19 desencadeou uma série de problemas que atingem não somente o setor de saúde pública como também a economia de diversos países afetados pela pandemia vigente. No Brasil, inúmeras tentativas de minimizar os efeitos da doença foram aplicadas e com isso, medidas preventivas contra a infecção foram adotadas, desde os grandes centros urbanos como também as indústrias e produção rural. O objetivo do presente estudo foi avaliar a adesão às práticas preventivas contra a COVID-19 no cenário do agronegócio da Região do Araguaia no Sudeste do Estado do Pará. O presente estudo caracteriza-se como exploratório com coleta de dados embasados na aplicação de um questionário visando verificar o grau de aceitação e confiabilidade das vacinas ofertadas em território nacional, na percepção de indivíduos que possuem atividades no agronegócio. Portanto, 50\% dos entrevistados ainda não conseguem ter uma resposta definitiva sobre a eficácia e segurança do imunizante, sendo que, $71,9 \%$ dos entrevistados apresentaram maior confiança em receber o imunizante desde que aprovado pelos órgãos regulamentadores nacionais. Quanto a máscara de proteção e uso de álcool em gel, 53,1\% e 68,7\% respectivamente utilizaram essas medidas preventivas durante toda a pandemia. $\mathrm{O}$ agronegócio sofre com as consequências da doença os dados obtidos no presente estudo apontam uma preocupação quanto a calamidade de saúde e economia gerada pela COVID-19, trazendo informações relevantes quanto ao perfil dos indivíduos atuantes no agronegócio da região estudada e suas escolhas quanto as metodologias preventivas ofertadas atualmente.
\end{abstract}

Palavras-chave: Agronegócio; Coronavírus; COVID-19; Praticas preventivas.

\begin{abstract}
The coronavirus that causes COVID-19 has triggered a series of problems that affect not only the public health sector but also the economy of several countries affected by the current pandemic. In Brazil, numerous attempts to minimize the effects of the disease were applied and with this, preventive measures against the infection were adopted, from the large urban centers as well as industries and rural production. The aim of the present study was to evaluate the adherence to preventive practices against COVID-19 in the agribusiness scenario of the Araguaia Region in the Southeast of the State of Pará. This study is characterized as exploratory with data collection based on the application of a questionnaire aiming to verify the degree of acceptance and reliability of vaccines offered in the national territory, in the perception of individuals who have activities in agribusiness. Therefore, $50 \%$ of respondents still cannot have a definitive answer on the efficacy and safety of the immunizing agent, and $71.9 \%$ of respondents showed greater confidence in receiving the immunizing agent since it was approved by the national regulatory bodies. As for the protective mask and the use of alcohol gel, $53.1 \%$ and $68.7 \%$ respectively used these preventive measures throughout the pandemic. Agribusiness suffers from the consequences of the disease. The data obtained in this study point to a concern about the health and economic calamity generated by COVID-19, bringing relevant information about the profile of individuals working in agribusiness in the region studied and their choices regarding methodologies preventive measures currently offered.
\end{abstract}

Keywords: Agribusiness; Coronaviruses; COVID-19; Preventive practices. 


\begin{abstract}
Resumen
El coronavirus que provoca el COVID-19 ha desencadenado una serie de problemas que afectan no solo al sector de la salud público sino también a la economía de varios países afectados por la pandemia actual. En Brasil se realizaron numerosos intentos de minimizar los efectos de la enfermedad y con ello se adoptaron medidas preventivas contra la infección, tanto de los grandes centros urbanos como de las industrias y la producción rural. El objetivo del presente estudio fue evaluar la adherencia a prácticas preventivas contra COVID-19 en el escenario agroindustrial de la Región Araguaia en el Sureste del Estado de Pará, este estudio se caracteriza por ser exploratorio con recolección de datos a partir de la aplicación de un cuestionario cuyo objetivo es verificar el grado de aceptación y confiabilidad de las vacunas que se ofrecen en el territorio nacional, en la percepción de los individuos que tienen actividades en el agroindustrial. Por lo tanto, el 50\% de los encuestados aún no puede tener una respuesta definitiva sobre la eficacia y seguridad del agente inmunizante, y el 71,9\% de los encuestados mostró una mayor confianza en recibir el agente inmunizante una vez aprobado por los organismos reguladores nacionales. En cuanto a la mascarilla protectora y el uso de gel de alcohol, el 53,1\% y el $68,7 \%$ respectivamente utilizaron estas medidas preventivas durante toda la pandemia. La agroindustria sufre las consecuencias de la enfermedad. Los datos obtenidos en este estudio apuntan a una preocupación por la calamidad sanitaria y económica que genera el COVID-19, aportando información relevante sobre el perfil de las personas que laboran en la agroindustria en la región estudiada y sus elecciones en materia de agroindustria. Metodologías de medidas preventivas que se ofrecen actualmente.
\end{abstract}

Palabras clave: Agronegocios; Coronavirus; COVID-19; Practicas preventivas.

\title{
1. Introdução
}

A pandemia da COVID-19 (do inglês Coronavirus Disease 2019), causada pelo SARS-CoV-2 (Coronavírus da Síndrome Respiratória Aguda Grave 2), tem gerado uma crise mundial que demanda estratégias emergenciais e ação coletiva para solucionar o problema pandêmico. (Camara, Pinto, Silva, 2020). Diante disto, observa-se efeitos que vão muito além do setor de saúde pública, mas que reflete em prejuízos consideráveis a economia de diversos países afetados pela doença.

No Brasil, além do número de mortos que aumentam exponencialmente, com todas as medidas preventivas empregadas por governos municipais ou federal, a queda do Produto Interno Bruto (IBGE) per capita em 2020 foi de 4,1\%, totalizando R\$ 7,4 trilhões, com redução significativa do número de empresas e aumento de desempregados (IBGE, 2020).

Apesar da queda do PIB per capita em 2020 no Brasil, foi possível observar que o PIB do agronegócio brasileiro teve alta de 2,2\% quando comparado ao ano anterior, representado uma parcela de 26,6\% no PIB brasileiro e 6 meses consecutivos de expansão, tendo uma safra recorde de grãos 2019/2020, a forte demanda internacional por produtos do agronegócio brasileiro e a taxa de câmbio influenciou este crescimento (CEPEA, 2020). A alta exportação de produtos agropecuários para países como a China $(34,1 \%)$ e Estados Unidos $(9,7 \%)$ garantiram um crescimento inesperado, visto os efeitos da pandemia do COVID-19 (Schneider et al., 2020).

Em contrapartida ao crescimento do PIB no agronegócio em 2020, os produtores rurais foram afetados de várias formas, como: a falta de protocolos de segurança e proteção sanitária que permitam aos agricultores trabalharem com tranquilidade, interagindo com a comunidade; dificuldades de logística, transporte, distribuição e comercialização dos alimentos; e restrições de acesso ao capital financeiro decorrentes dos efeitos da pandemia nas economias nacionais (IICA, 2020).

Dentre as diferentes medidas de prevenção aprovadas pela Organização Mundial da Saúde (OMS), as mais recomendadas para o combate a COVID-19 foram a higienização das mãos com água e sabão com posterior aplicação de álcool em gel, a utilização de máscaras, o distanciamento social, além dos decretos governamentais aplicados para a redução da disseminação da doença em ambiente urbano e rural.

No entanto, a aceitação das medidas preventivas pela população ainda é vista como um desafio, visto que uma parte considerável dos cidadãos não creem na eficiência dessas medidas ou minimizam os efeitos que a infecção pelo vírus pode causar (Buntin, 2020). No geral, os comportamentos preventivos individuais frente às epidemias podem ser influenciados por fatores psicossociais, demográficos e comportamentos em saúde. O uso de máscaras em locais públicos e o hábito de lavar as mãos frequentemente, por exemplo, foram mais comuns entre mulheres, indivíduos mais velhos, com maior nível 
socioeconômico, com menores níveis de estresse, ansiedade e depressão e entre aqueles com maior percepção sobre a severidade da doença (Zhong et al; Bish; Machida et al.; Wang et al.; Muto et al., 2020).

Tendo em vista a alta demanda pela busca de soluções para o enfrentamento da pandemia vigente, é possível observar uma resistência por parte de alguns indivíduos quanto ao uso de medidas preventivas, visto que em muitos casos essas pessoas são descrentes quanto a efetividade das mesmas no combate do vírus. A população rural, na maior parte das vezes, carece de informações pertinentes em relação ao mecanismo de infecção ou a eficácia das formas de prevenção, além do fato de que muitas famílias atuantes no agronegócio sofrem diariamente pela falta de profissionais da saúde atuantes no zonal rural, dada a alta demanda destes na linha de frente ao combate do vírus nos grandes centros urbanos (Silva, 2020). Poucos estudos são encontrados na literatura tendo como foco observar os impactos sociais, econômicos, psicológicos ou na saúde dos indivíduos envolvidos no agronegócio, além de ser notável uma carência de informações quanto ao posicionamento dessas pessoas frente ao grave problema enfrentado.

Portanto, dada a justificativa e relevância do presente estudo, o mesmo tem como objetivo avaliar a adesão dos atuantes do agronegócio às práticas preventivas contra a COVID-19 na Região do Araguaia no Sudeste do Estado do Pará.

\section{Metodologia}

\section{Características do local de estudo}

Localizada na Região Sudeste do Pará e entrecortada pelas rodovias BR-158 e PA-279, a Região de Integração Araguaia detém uma área territorial de mais de 147 mil quilômetros quadrados, o que representa 14\% da área total do Pará. Nas últimas décadas a extração madeireira, a pecuária bovina e a extração mineral têm sido suas principais atividades produtivas.

\section{Característica da aplicação do estudo}

O presente estudo caracteriza-se como exploratório com coleta de dados embasados na aplicação de um questionário tendo como tema principal as medidas preventivas contra a COVID-19 e sua aceitação no agronegócio. O documento foi disponibilizado via plataforma online, dado a impossibilidade de aplicação presencial, respeitando o momento da pandemia. Os questionários foram aplicados em um grupo de interesse, relacionados a produção rural na Região do Araguaia no Sudeste do Estado do Pará: Gestores e colaboradores atuantes na pecuária, agricultura, transporte de alimentos e insumos e nas agroindústrias (grãos e carne bovina).

Os questionários foram aplicados no período de 28 de janeiro a 05 de março de 2021. As perguntas tinham interesse em avaliar o grau de aceitação e confiabilidade de indivíduos envolvidos no agronegócio com relação as medidas preventivas relacionadas a COVID-19. Ao todo 12 perguntas (11 objetivas, 01 discursiva) foram elaboradas, sendo 05 associadas a informações pessoais como: nome, cidade, escolaridade, gênero e ramo do agronegócio envolvido. Os respondentes foram orientados quanto aos riscos da pesquisa, sendo abertamente mencionado que a desistência dos mesmos poderia ser solicitada a qualquer momento. É importante mencionar que nenhuma informação pessoal, seja nome ou meios de contato foram ou serão divulgadas, garantindo o sigilo dos mesmos, sendo os respondentes avisados sobre isso também.

\section{Critérios de elegibilidade}

O presente estudo teve como principais critérios para a seleção de respondentes: indivíduos que, independentemente de gênero, tinham envolvimento total em atividades voltadas ao agronegócio na região Araguaia no Sudeste do Estado do Pará, ter idade maior ou igual a 18 anos e ter concordado e acordado com todos os critérios impostos pelo Termo de Consentimento Livre e Esclarecido (TCLE) apresentados a eles no momento da pesquisa. 
Não foram aceitos neste estudo a resolução do questionário por mais de um (1) membro do mesmo grupo familiar, sendo restrito a participação para apenas um integrante da família, dado a preferência a aquele que estivesse mais fortemente envolvido a atividades seja, na produção e/ou distribuição de produtos relacionados ao agronegócio regional e/ou pesquisadores das ciências agrárias.

\section{Resultados e Discussão}

Ao todo 32 indivíduos respeitaram os critérios de elegibilidade do presente estudo e participaram na resolução do questionário. As características dos respondentes podem ser observadas na Tabela 1. Em relação a qual grupo dentro do agronegócio cada uma estava envolvido: 40,6\% (13/32) caracterizavam-se como proprietário de estabelecimentos rurais, 40,6\% (13/32) atuavam como colaboradores em fazendas ou sítios da região, 12,5\% (4/32) eram funcionários do ramo industrial, trabalhando em silos e frigoríficos e 6,2\% (2/32) eram envolvidos exclusivamente no transporte de alimentos e insumos na região.

Tabela 1 - Distribuição das características pessoais dos entrevistados $(\mathrm{n}=32)$

\begin{tabular}{lr}
\hline Faixa etária & 17 \\
18 a 25 anos & 10 \\
26 a 40 anos & 2 \\
41 a 60 anos & 3 \\
Acima dos 60 anos & \\
\hline Gênero & 23 \\
Masculino & 9 \\
Feminino & \\
Grau de escolaridade & 9 \\
Ensino Superior Incompleto & 7 \\
Ensino Superior Completo & 6 \\
Ensino Fundamental Incompleto & 3 \\
Ensino Fundamental Completo & 1 \\
Ensino Médio Incompleto & 4 \\
Ensino Médio Completo & 2 \\
\hline Não alfabetizado
\end{tabular}

Fonte: Autores.

Quanto o grau de escolaridade associada a intenção de receber o imunizante, dos 16 indivíduos incluídos no ensino superior (completo ou incompleto), $81 \%$ receberiam o imunizante, conforme observado nos dados obtidos através do questionário. Entretanto, apenas $40 \%$ (2/5) dos indivíduos no ensino médio (completo e incompleto) receberiam a vacina. Dados ainda destacam que, 66,6\% (6/9) dos participantes que possuem o ensino fundamental como grau de escolaridade também se mostraram adeptos a essa metodologia de prevenção, visto também que isso ocorreu com todos os indivíduos categorizados como não alfabetizados.

Os resultados da análise de escolaridade se assemelham com outros estudos realizados em âmbito nacional, seguindo as mesmas tendências com relação a comparação da aceitação da vacina quanto ao grau de escolaridade dos indivíduos. Segundo estudos do Datafolha (2021) realizados com 2.016 brasileiros, os que estudam até o ensino fundamental o percentual de vacinação é de $81 \%$ contra $86 \%$ dos que concluíram o ensino superior. 
Um grupo importante que se destacou no presente estudo foi os não alfabetizados que os dois indivíduos entrevistados se mostraram adeptos a receber o imunizante, mesmo não tendo conhecimento científico quanto a composição e princípio de ação da vacina. Na tentativa de justificar essa aceitação, pode-se mencionar o impacto da mídia que diariamente traz atualizações nos números de indivíduos afetados e óbitos da doença, além de contribuir ativamente para o incentivo, na maior parte das vezes, a vacinação.

O presente estudo foi realizado na região do Araguaia no Sudeste do Estado do Pará. A Figura 1 representa as cidades onde os envolvidos no questionário residiam e atuavam profissionalmente. A região Araguaia no Pará é conhecida pelo seu potencial em produção agrícola em especial atividades de pecuária de corte e exportação de grãos. 46,9\% (15/32) dos participantes eram da cidade de Redenção do Pará, local este onde observa-se um crescente aumento do número de infectados.

Figura 1: Distribuição do número de entrevistados em cidades pertencentes a região do Araguaia no Estado do Pará.

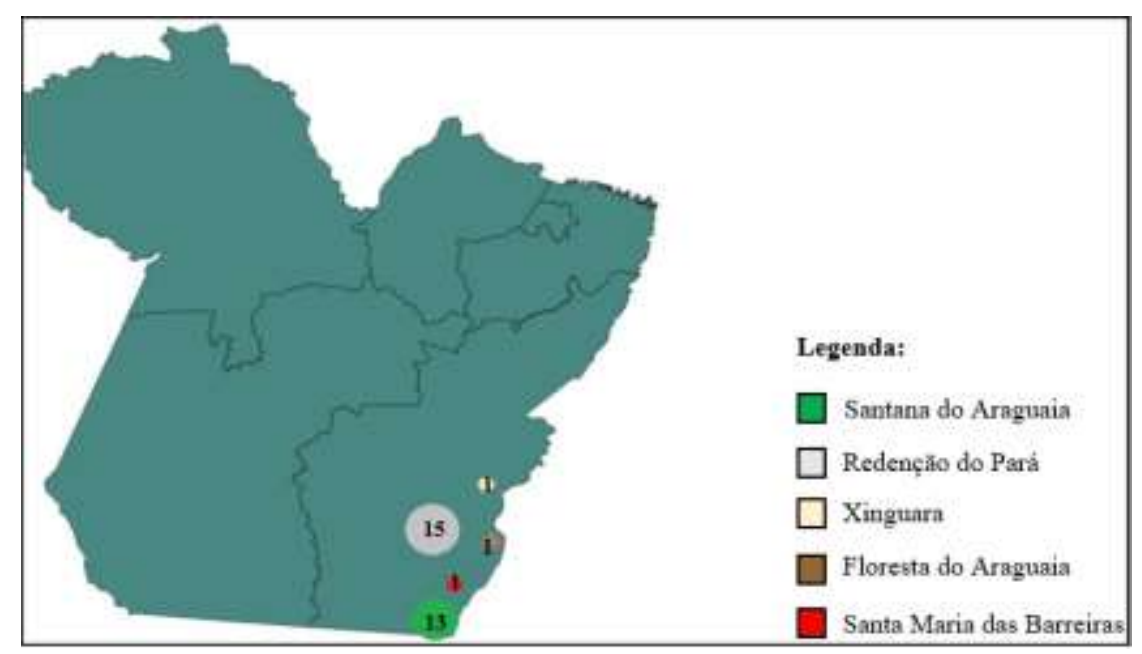

Fonte: Autores.

Uma das principais investigações realizadas através da aplicação do questionário foi verificar o grau de aceitação e confiabilidade das vacinas ofertadas em território nacional, na percepção de indivíduos que possuem atividades no agronegócio. Conforme observado na tabela 2, 50\% (16/32) dos entrevistados ainda não conseguem ter uma resposta definitiva sobre a eficácia e segurança do imunizante. Segundo De Figueiredo et al. (2020) O Brasil figura entre os países onde mais aumentou a desconfiança no sistema de imunização, trazendo a diminuição de $73 \%$ para $63 \%$ nos que creem que as vacinas são seguras.

Umas das grandes problemáticas associadas a vacina é a dúvida que muitas pessoas possuem, incluindo os entrevistados deste estudo, quanto a rapidez na produção da vacina. Segundo Pronker et al. (2013) uma vacina normalmente requer um cronograma que dura em média 10 anos de duração, o que não foi observado com as vacinas contra a COVID-19, sendo a maior parte produzidas em menos de 01 ano dos primeiros relatos da doença. No entanto, Hotez et al. (2021) justifica que as pesquisas envolvendo o vírus não são recentes, e sim cerca de 10 anos de estudos genômicos resultando numa maior rapidez na produção do imunizante contra o novo coronavírus, Além dos inúmeros aliados políticos e científicos unidos por um objetivo em comum, diminuindo a burocratização da vacina em questão. 
Tabela 2 - Avaliação do grau de confiabilidade da vacina contra COVID-19 em indivíduos associados ao agronegócio.

\begin{tabular}{cccc}
\hline Perguntas & & \multicolumn{2}{c}{ Frequência absoluta } \\
\hline $\begin{array}{c}\text { Você acha que as vacinas contra a COVID-19 disponibilizadas em } \\
\text { território nacional são seguras e eficazes? }\end{array}$ & Sim & Não & Não sei responder ainda \\
Você receberia a vacina contra a COVID-19 se soubesse que a eficácia \\
foi comprovada cientificamente e que foi aprovada pelos órgãos \\
regulamentares nacionais (ANVISA)?
\end{tabular}

Fonte: Autores.

Mesmo a maior parte dos entrevistados não terem uma resposta concreta a respeito da disponibilização das vacinas, sua segurança e eficácia, 71,9\% (23/32) dos entrevistados apresentaram maior confiança em receber o imunizante desde que aprovado pelos órgãos regulamentadores nacionais.

Outra avaliação importante para o presente estudo, foi verificar como os respondentes se comportaram com relação ao uso das medidas preventivas aprovadas e recomendadas pela OMS, como observado na tabela 3. Quanto a máscara de proteção e uso de álcool em gel, foi possível observar que entre os respondentes $53,1 \%$ e $68,7 \%$ respectivamente utilizaram essas medidas preventivas durante toda a pandemia. No entanto, observa-se um aumento no número de indivíduos que fazem uso de álcool para higienização das mãos e superfícies quando comparada com a utilização de máscaras de proteção, mesmo com a vacina sendo ofertada. Uma das justificativas mais esclarecedoras é a comodidade na qual essa medida permite, pois muitos respondentes associam a máscara a um desconforto considerável, atrapalhando nos movimentos respiratórios, refletindo em dificuldades no trabalho diário.

Estudos anteriores como o de Clements e Lee e You (2020) mostraram ampla variação na adoção dessas medidas em diferentes populações, durante a pandemia da COVID-19. O uso de máscaras em locais públicos foi reportado por 23,6\% dos adultos nos Estados Unidos, 63,2\% na Coreia do Sul, já Huang et al. (2020) observou uma frequência muito maior com cerca de $98 \%$ de adesão na China.

Tabela 3. Avaliação do comportamento dos respondentes em associação as medidas de prevenção recomendadas contra a COVID-19

\begin{tabular}{|c|c|c|c|c|c|c|}
\hline \multirow{2}{*}{$\begin{array}{c}\text { Medida } \\
\text { preventiva }\end{array}$} & \multicolumn{6}{|c|}{ Frequência absoluta } \\
\hline & $\begin{array}{c}\text { Utilizei / Mantive } \\
\text { durante toda a } \\
\text { pandemia e continuo } \\
\text { utilizando mesmo com } \\
\text { a vacina já sendo } \\
\text { ofertada }\end{array}$ & $\begin{array}{l}\text { Não utilizei / } \\
\text { mantive durante } \\
\text { toda a pandemia, } \\
\text { somente quando } \\
\text { exigido em algum } \\
\text { estabelecimento }\end{array}$ & $\begin{array}{l}\text { Utilizei / Mantive } \\
\text { durante toda a } \\
\text { pandemia e continuo } \\
\text { utilizando mesmo } \\
\text { depois de infectado }\end{array}$ & $\begin{array}{l}\text { Utilizei/ Mantive } \\
\text { apenas no início da } \\
\text { pandemia e parei } \\
\text { de usar mesmo não } \\
\text { sendo infectado } \\
\text { pelo vírus }\end{array}$ & $\begin{array}{l}\text { Utilizei / } \\
\text { Mantive no } \\
\text { início da } \\
\text { pandemia e } \\
\text { parei de usar } \\
\text { após a infecção } \\
\text { pelo vírus }\end{array}$ & $\begin{array}{l}\text { Utilizei / Mantive } \\
\text { durante toda a } \\
\text { pandemia, mas não } \\
\text { continuarei } \\
\text { utilizando após ser } \\
\text { vacinado }\end{array}$ \\
\hline $\begin{array}{l}\text { Máscara de } \\
\text { proteção }\end{array}$ & 13 & 10 & 4 & 3 & 1 & 1 \\
\hline Álcool em gel/ $70^{\circ}$ & 19 & 6 & 3 & 3 & 1 & - \\
\hline $\begin{array}{l}\text { Distanciamento } \\
\text { social }\end{array}$ & 14 & - & 2 & 9 & 7 & - \\
\hline
\end{tabular}


Embora não tenham sido ainda identificados, até o presente momento, estudos para avaliar o grau de adesão da população brasileira a essas medidas preventivas dentro do campo do agronegócio, segundo Datafolha (2020) 76\% são favoráveis à manutenção do distanciamento social para controlar a pandemia, mesmo que isso signifique prejuízos econômicos. No estudo de Lyu e Wehby (2020), avaliando a eficácia dos decretos governamentais no enfretamento a pandemia, foi possível observar que o uso de máscaras faciais em público resultou num declínio maior nas taxas de crescimento diário do COVID-19 após a emissão dos decretos, em comparação com os estados que não emitiram.

Os resultados quanto a adesão ao distanciamento social no presente estudo, podem ter sido influenciados devido a diferentes classes de entrevistados, em especial os proprietários e colaboradores rurais (81,2\%) que de certa forma, involuntariamente evitaram aglomerações, devido a distância de seu local de trabalho com relação aos centros urbanos, onde ocorrem uma maior taxa de agrupamento de pessoas. Independente da oferta de vacina ou infecção pregressa 50\% (16/32) dos entrevistados se mostraram adeptos e mantiveram/mantém o distanciamento social. Outro fato observado foi o posicionamento dos entrevistados que mantiveram esta metodologia preventiva apenas no início da pandemia ou até o momento em que foram infectados.

Por fim, uma última análise foi empregada afim de entender o principal motivo na qual os respondentes respeitaram/ respeitam as medidas preventivas contra a COVID-19. De forma descritiva, 75\% dos entrevistados (24/32) alegam que a proteção ao grupo de risco, incluindo familiares que podem sofrer maiores impactos na infecção, torna-se a principal justificativa para esse posicionamento, a garantia do cumprimento dos decretos governamentais novamente foi levantada. Quando considerado o isolamento social é importante destacar que os entrevistados se mostraram adeptos a sair de casa em situações como ida a mercado, farmácia ou prática de atividades físicas. Entre os idosos, por exemplo, segundo o estudo de Malta et al (2020) a prevalência da prática de atividade física reduziu de 30,4\% para 14,2\%. No entanto, o estudo de Lesser e Nienhuis (2020) conduzido no Canadá observou que indivíduos ativos reportavam maiores níveis de atividade física durante a pandemia, em comparação ao grupo dos inativos. Não foram encontrados estudos avaliando a aceitação do distanciamento e/ou isolamento social com os indivíduos residentes no ambiente rural, em especial na região Norte do país.

\section{Conclusão}

A pandemia vigente do COVID-19 trouxe consequência na saúde e economia em todo o país. O agronegócio independente do crescimento apontado em 2020 sofre com as consequências da doença, devido ao risco que uma boa parte dos trabalhadores estão sendo exposto diariamente, em especial, aqueles que apresentam comorbidades. $\mathrm{O}$ presente estudo demostrou que $50 \%$ dos entrevistados se mostraram duvidosos quanto a segurança e eficácia da vacina, no entanto, 71,9\% se mostraram confiantes em receber o imunizante quando aprovado pelos órgãos regulamentadores nacionais.

Além disso, o estudo foi esclarecedor quanto ao posicionamento dos indivíduos atuantes no agronegócio em relação às metodologias preventivas recomendadas, observando que a maior parte dos entrevistados utilizaram/utilizam álcool em gel e máscara durante a pandemia vigente. Quanto ao distanciamento social somente metade dos entrevistados aderiram/aderem durante o prosseguimento da pandemia.

Os dados obtidos no presente estudo apontam uma preocupação quanto a calamidade de saúde e economia gerada pela COVID-19, trazendo informações relevantes quanto ao perfil dos indivíduos atuantes no agronegócio da região do Araguaia no Sudeste do Estado do Pará e suas escolhas quanto as metodologias preventivas ofertadas atualmente. Ainda é preocupante a relutância de algumas pessoas a aceitação da magnitude do problema vigente, em especial os envolvidos em atividades rurais, nas quais foram o foco do presente estudo. Entender o vírus e as formas de como evitar o contato com o mesmo, é uma peça fundamental para a redução dos números de casos e consequentes óbitos que são relatados e que nos distanciam cada vez mais de um quadro mais positivo, mesmo com vacinas já sendo ofertadas em todo o território nacional. 
Poucos dados são encontrados atualmente na literatura, quanto ao posicionamento dos indivíduos em relação as medidas preventivas, em especial no Estado do Pará. Se faz necessário novos estudos, com maior número amostral e que elucidem melhor a tendência da população a aderir essas metodologias de combate e prevenção ao COVID-19.

\section{Referências}

Bish, A., \& Michie, S. (2010). Demographic and attitudinal determinants of protective behaviours during a pandemic: a review. British Jorunal of Health Psychology, 15, 797-824.

Buntin, M. B. (2020). Social Distancing-What Would It Mean for It to Work? https://jamanetwork.com/channels/health-forum/fullarticle/2764778

Câmara, S. F., Pinto, F. R., Silva, F. R., \& Gerhard, F. (2020) Vulnerabilidade socioeconômica à COVID-19 em municípios do Ceará. Revista de Administração Pública, 54 (4), 1037-1051.

Centro de Estudos Avançados em Economia Aplicada - CEPEA. (2020). https://www.cepea.esalq.usp.br/br/pib-do-agronegocio-brasileiro.aspx

Clements J. M. (2020). Knowledge and behaviors toward COVID-19 among us residents during the early days of the pandemic: cross-sectional online questionnaire. JMIR Public Health Surveillance, 6: e19161.

De Figueiredo, A., Simas, C., Karafillakis, E., Paterson, P., \& Larson, H. (2020) Mapping global trends in vaccine confidence and investigating barriers to vaccine uptake: a large-scale retrospective temporal modelling study. Lancet, 396 (10255), 898-908.

Hotez, P., Batista, C., Ergonul, O., Figueroa, J. P., Gilbert, S., Gursel, M., Hassanain, M., Kang, G., Kim, J. H., Lall, B., Larson, H., Naniche, D., Sheahan, T., Shoham, S., Wilder-Smith, A., Strub-Wourgaft, P. Y., \& Botazzi, M. E. (2021). Correcting COVID-19 vaccine misinformation. Eclinical Medicine, 33 (100780).

Huang, Y., Wu, Q., Wang, P., Xu, Y., Wang, L., Zhao, Y., et al. (2020). Measures undertaken in China to avoid COVID-19 infection: internet-based, crosssectional survey study. Journal of Medical Internet Research, 22: e18718.

Instituto Brasileiro de Geografia e Estatísticas - IBGE. Estatística econômicas: PIB brasileiro e associação a pandemia de COVID-19 (2020). https://agenciadenoticias.ibge.gov.br/agencia-sala-de-imprensa/2013-agencia-de noticias/releases/30165-pib-cai-4-1-em-2020-e-fecha-o-ano-em-r-7-4-trilhoes

Instituto Interamericano de Cooperação para a Agricultura - IICA. La agricultura familiar y el abastecimiento agroalimentario ante la pandemia COVID-19 en América Latina y el Caribe. Programa de Desarrollo Territorial y Agricultura Familiar. (2020). https://www.iica.org.br/pt/covid19

Lee M., \& You M. (2020). Psychological and behavioral responses in South Korea during the early stages of coronavirus disease 2019 (COVID-19). International Journal of Environmental Research and Public Health, 17:2977.

Lesser, I. A., \& Nienhuis, C. P. (2020). The impact of COVID-19 on physical activity behavior and well-being of canadians. International Journal of Environmental Research and Public Health, 17:3899.

Lyu, W., \& Wehby, G. L. (2020). Community use of face masks and COVID-19: evidence from a natural experiment of state mandates in the US. Health Affairs, 39 (1), 1419-1425.

Machida, M., Nakamura, I., Saito, R., Nakaya, T., Hanibuchi, T., Takamjya, T, et al. (2020). Changes in implementation of personal protective measures by ordinary japanese citizens: a longitudinal study from the early phase to the community transmission phase of the COVID-19 outbreak. International Journal of Infectious Diseases, 96, 371-375.

Malta, D. C., Szwarcwald, C. L., Barros, M. B. A., Gomes, C. S., Machado, I. E., \& Souza-Júnior, P. R. B. (2020). The COVID-19 pandemic and the changes in adult Brazilian lifestyles: a cross-sectional study, 2020. Epidemiologia e Serviços de Saúde, 29: e2020407.

Muto, K., Yamamoto, I., Nagasu, M., Tanaka, M., \& Wada, K. (2020). Japanese citizens' behavioral changes and preparedness against COVID-19: an online survey during the early phase of the pandemic. PLoS One, 15: e0234292.

Pronker E. S., Weenen T. C., Commandeur H., Claassen E. H. J. H. M., \& Osterhaus, A. D. M. E. (2013). Risk in vaccine research and development quantified. PLOS ONE, 8 (3), 57755-57759.

Silva, B. N., \& Pinto, E. S. G. (2020). Saúde rural em tempos de pandemia da covid-19. Revista Cuidarte, 11 (3) :e1265

Schneider, S., Cassol, A., Leonardi, A., \& Marinho, M. (2020). Os efeitos da pandemia da COVID-19 sobre o agronegócio e a alimentação. Estudos Avançados, 34 (100), 167-188.

Wang, C., Pan, R., Wan, X., Tan, Y., Xu, L., Ho, C. S, et al. (2020) Immediate psychological responses and associated factors during the initial stage of the 2019 coronavirus disease (COVID-19) epidemic among the general population in China. International Journal of Environmental Research and Public Health, 17,1729 .

Zhong, B. L., Luo, W., Li, H. M., Zhang, Q. Q., Liu, X. G., Li, W. T., et al. (2020). Knowledge, attitudes, and practices towards COVID-19 among chinese residents during the rapid rise period of the COVID-19 outbreak: A quick online cross-sectional survey. International Journal of Biological Sciences, 16, $1745-1752$. 